\section{Intestinal-Allergie: Diagnostik direkt aus dem Darm?}

\section{Neben dem plazebokontrollierten oralen Provokationstest gibt es heute elegante Möglichkeiten, eine intestinale Allergie direkt am Ort des Geschehens dingfest zu machen. Werkzeuge sind die Endoskopie und die gastrointestinale Lavage.}

Läßt die Anamneseerhebung und die Auswertung eines Ernährungstagebuchs die Verdachtsdiagnose einer Nahrungsmittelallergie zu, kann man guten Gewissens erst bei einer weitergehenden Diagnostik dem Patienten Therapieempfehlungen z.B. in Hinsicht Allergenkarenz geben.

Aber auch RAST- und Hauttests sind nach den Worten von M. Raithel, Erlangen, gerade bei erwachsenen $\mathrm{Pa}-$ tienten mit einer gastrointestinalen Allergie wenig aussagekräftig. Einige Patienten mit einer lokal begrenzten gastrointestinalen Allergie zeigen sogar überhaupt keine Hautreaktion. Für eine präzise Diagnosenstellung gilt der doppelblind und plazebokontrolliert durchgeführte orale Provokationstest nach wie vor als Goldstandard.

\section{Methylhistamintest als \\ Eingangsuntersuchung}

Doch lassen es moderne Diagnosemethoden der Gastroenterologie heute zu, sich direkt an den Ort des Geschehens zu begeben. In Erlangen, so Raithel, wird bei Patienten mit Verdacht auf eine Intestinal-Allergie zunächst ein Routinetest auf Methylhistamin - einem Hauptabbauprodukt des Histamins - im Sammelurin durchgeführt. Ein erhöhter Methylhistamingehalt zeigt an, ob überhaupt eine histaminbedingte Erkrankung vorliegt.

Bei positivem Resultat wird der Patient einer Endoskopie unterzogen, möglichst an einem Tag, an dem er gastrointestinale Beschwerden hat. „Die Endoskopie dient der Quantifizierung

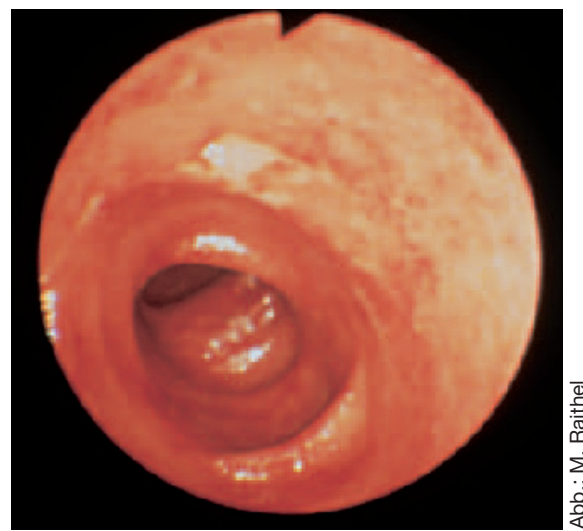

Gastrointestinale Lebensmittelallergie durchs Endoskop betrachtet: Nach Allergenprovokation kommt es zu petechialen Einblutungen und Erosionen der Darmschleimhaut.

der intestinalen allergischen Entzündungsreaktion“, so Raithel. Liegt ein allergisches Geschehen vor, erkennt man feinfleckige Erytheme und - bei stärkeren Reaktionen - petechiale Einblutungen (s. Abb.).

an Gewebeproben des Darms

Gleichzeitig können während der Endoskopie Gewebeproben entnommen werden, in denen der Gehalt an spezifischen Allergiemarkern gemessen wird. Auch kann man an den Biopsien eine Ex-vivo-Allergenstimulation vornehmen. Bei diesem sog. Mukosa-Oxygenationstest werden die Biopsien in einem Kulturmedium unter Sauerstoffbegasung 1 bis 2 Tage am Leben gehalten und mit Allergenen stimuliert. Bei

\section{Ex-vivo-Allergenstimulation}

einer positiven Reaktion schütten die Mastzellen und eosinophilen Granulozyten der Biopsie Histamin, ECP (Eosinophiles kationisches Protein) und Tryptase aus, deren Konzentrationsanstieg im Kulturmedium gemessen wird.

Eine weitere Möglichkeit besteht in der endoskopischen Lavage von Dünndarm und vor allem terminalem Ileum, in dem sich die meisten immunkompetenten Zellen befinden. In der Spülflüssigkeit können spezifische, lokal in dem betreffenden Segment gebildete IgE-Antikörper nachgewiesen werden.

\section{Kombination nicht-medikamentöser und medikamentöser Verfahren}

Hat sich der Verdacht auf eine Nahrungsmittelallergie erhärtet, wird je nach Schwere- bzw. Ausbreitungsgrad der Allergie therapiert. Basis ist die Allergenkarenz (soweit möglich) zusammen mit der Zufuhr hypoallergener Kostformen mit Oligopeptid- und Aminosäurepräparaten. Zusätzlich sollte die Nahrung wenig Histamin enthalten.

Medikamentös wird das Dinatriumsalz der Cromoglicinsäure (DNCG) gegeben. Diese Basistherapie ist ausreichend, wenn sich die nur leicht ausgeprägte und intermittierend auftretende Allergie auf Mukosa und Submukosa des Gastrointestinaltrakts (GI-Trakt) beschränkt. Etwa 55\% der Patienten, so Raithel, lassen sich mit dieser Kombination von medikamentösen und nicht-medikamentösen Therapieverfahren befriedigend behandeln.

Ist die Symptomatik stärker und sind zunehmend extraintestinale Organe in Mitleidenschaft gezogen, so wird zusätzlich Ketotifen gegeben. Reicht das nicht aus - gerade, wenn zusätzlich Urtikaria, Rhinokonjunktivitis oder bronchiale Symptome bestehen - sollten nicht-sedierende Antihistaminika wie Cetirizin, Loratadin oder Fexofenadin zusätzlich zu Ketotifen verabreicht werden, so Raithel.

bk

Anmerkung der Redaktion: Dieser Artikel gibt nicht die Meinung der Herausgeber des Allergo Journal wieder.

Vortrag von M. Raithel, Erlangen, anläßlich des CXXXVI. Practicum Allergologicum des Ârzteverbandes Deutscher Allergologen, Regionalgruppe Westfalen Lippe, Münster 1999. 